\title{
Patología placentaria y riesgo perinatal durante la pandemia por COVID-19
}

Víctor M. Vargas-Hernández*, Jesús E. Luján-Irastorza y Carlos Durand-Montaño

Clínica PRONATAL (Bité Médica Hospital), Ciudad de México, México

\section{Resumen}

Antecedentes: Los cambios respiratorios e inmunitarios en el embarazo pueden conducir a infecciones virales. En la enfermedad por coronavirus 2019 (COVID-19) las características clínicas y riesgos perinatales son difíciles de evaluar y son relativamente desconocidos. Objetivo: Revisar patología placentaria en mujeres asintomáticas con COVID-19 y evaluar efectos en datos perinatales. Material y método: Estudio retrospectivo, observacional y transversal, incluye 29 mujeres embarazadas en 2020. Se realizaron prueba COVID-19 y dividieron en dos grupos: 1) control, pacientes COVID-19 negativas y 2) COVID-19 asintomáticas con COVID-19 positivo; las placentas se estudiaron en patología y los datos clínicos se tomaron del expediente electrónico; asimismo, se realizó revisión de literatura. Resultados: Al comparar grupos no se observó diferencia en datos generales y características clínicas. El día del parto, las pacientes 2, 4, 5, 6, 8 y 9 del grupo COVID-19 se encontraban entre día 0 y 10.5 después de positividad; únicamente las pacientes 1, 3 y 7 habían superado la infección. Se presentó disminución de semanas de gestación en el grupo COVID-19 (37.8 \pm 1.8 vs. $39 \pm 0.8 ; p \leq 0.05)$. La histopatología placentaria en COVID-19 mostró mayor prevalencia de alteraciones trombóticas en vellosidades placentarias (55.5 vs. 0\%). Conclusiones: $\mathrm{La}$ infección asintomática por COVID-19, potencializa perfil protrombótico preexistente, incrementando riesgo de trombosis placentaria y trombosis en mujeres embarazadas.

PALABRAS CLAVE: COVID-19. Embarazo. Placenta. Trombosis.

\section{Placental pathology and perinatal risk during the COVID-19 pandemic}

\begin{abstract}
Background: Respiratory and immune changes during pregnancy can lead to viral infections. In coronavirus disease 2019 (COVID-19), clinical characteristics and perinatal risks are difficult to assess and are relatively unknown. Objective: To review placental pathology in asymptomatic women with COVID-19, and to evaluate effects on perinatal outcomes. Material and method: Retrospective, observational, cross-sectional study that included 29 pregnant women in 2020. The women underwent COVID-19 tests and were divided in two groups: 1) control, COVID-19-negative patients, and 2) asymptomatic COVID-19-positive patients; the placentas were studied at the pathology department, and clinical data were retrieved from the electronic medical record; in addition, a literature review was carried out. Results: When the groups were compared, no differences were observed in general data and clinical characteristics. On the day of delivery, patients 2, 4, 5, 6, 8 and 9 of the COVID-19 group were between day 0 and 10.5 after having tested positive; only patients 1,3 and 7 had overcome the infection. There was a decrease in weeks of gestation in the COVID-19 group (37.8 \pm 1.8 vs. $39 \pm 0.8 ; p \leq 0.05$ ). COVID-19-positive patients' placental histopathology showed a higher prevalence of thrombotic alterations in placental villi (55.5 vs. $0 \%$ ). Conclusions: COVID-19 asymptomatic infection potentiates preexisting prothrombotic profile, thus increasing the risk of placental thrombosis and, potentially, of thrombosis in pregnant women.
\end{abstract}

KEY WORDS: COVID-19. Pregnancy. Placenta. Thrombosis.

Correspondencia:

*Víctor M. Vargas Hernández

E-mail: vvargashernandez@yahoo.com.mx
Gac Med Mex. 2021;157:512-518

Disponible en PubMed

www.gacetamedicademexico.com

0016-3813/@ 2021 Academia Nacional de Medicina de México, A.C. Publicado por Permanyer. Este es un artículo open access bajo la licencia CC BY-NC-ND (http://creativecommons.org/licenses/by-nc-nd/4.0/). 


\section{Antecedentes}

La enfermedad por coronavirus 2019 (COVID-19) es una enfermedad respiratoria causada por el nuevo coronavirus 2 del síndrome respiratorio agudo grave (SARS-CoV-2), virus de ARN más contagioso e infeccioso que el SARS-CoV (2002) y el coronavirus causante del síndrome respiratorio de Oriente Medio (MERS-CoV) (2012) ${ }^{1-4}$.

Infecta células epiteliales a través de la enzima convertidora de angiotensina 2 (ACE2), empleando proteína Spike ${ }^{5-7}$. Genera síntomas como fiebre (83$98 \%)$, tos $(46-82 \%)$, mialgia o fatiga (11-44\%), disnea $(31 \%)$, gastropatías (10\%), hipotensión $(60 \%)^{6}$, linfopenia, leucopenia, trombocitopenia, incremento de citocinas inflamatorias, biomarcadores cardiacos elevados y disminución de albúmina ${ }^{6}$. Aunque las enfermedades respiratorias por COVID-19 son foco de atención, existen estudios que reportan lesiones oculares ${ }^{7}$, alteraciones testiculares ${ }^{8}$, ACE2 en ovario ${ }^{9}$, alteraciones de sistema nervioso ${ }^{10,11}$, trombosis ${ }^{12}$, alteraciones placentarias ${ }^{13}$ y complicaciones en embarazo ${ }^{14,15}$.

Por lo anterior, analizamos una serie de casos de embarazadas con COVID-19 asintomáticas, con el objetivo de analizar datos histopatológicos placentarios, para evaluar efectos y riesgos fetales en último trimestre del embarazo, aunado a revisión de literatura reciente.

\section{Material y método}

Estudio retrospectivo, observacional y transversal, que incluye a 29 pacientes que acudieron en 2020 a la clínica PRONATAL (Hospital Bíte Médica, CDMX) para atención obstétrica. Los datos clínicos se tomaron del expediente electrónico, previo consentimiento informado.

Las pacientes fueron hospitalizadas en la sala de labor para atención obstétrica, siguiendo protocolo implementado por pandemia de COVID-19. Incluyó presentación de prueba COVID-19, no mayor a una semana antes de realizarse el ingreso de paciente o en caso contrario al ingreso, si presentaban complicaciones como amenaza de parto prematuro. El personal de salud portaba material de seguridad para evitar contagios (cubrebocas, caretas, gorros, guantes, etc.) y el quirófano se desinfectaba antes y después de su utilización ${ }^{16}$. Se formaron 2 grupos: 1) control, las que nunca adquirieron COVID-19 y 2) COVID-19, las asintomáticas con COVID-19 en tercer trimestre de gestación.
Las pruebas de COVID-19 se enviaron al Centro de Diagnóstico Celular (Genes and Care, Ciudad de México), reacción en cadena de la polimerasa con transcriptasa inversa (RT-PCR), kit de detección: Logix Smart TM Coronavirus Disease 2019 (COVID19), Validación InDRE oficio DGE-DSAT-03863-2020. Permiso InDRE oficio DGE-DDYR-08355-2020.

Las placentas se procesaron por el Departamento de Patología Bíte Médica. Las secciones típicas se fijaron en formalina, se procesaron en parafina y tiñeron con hematoxilina/eosina.

La recolección de datos se realizó por enfermería, médicos e investigador de PRONATAL (datos generales, resultados COVID-19 e histopatología placentaria). Protegiendo el anonimato, sin referenciar origen de información y solo mostrando datos numéricos. Criterios de inclusión: mujeres asintomáticas positivas a COVID-19, embarazos a término y pretérmino. Criterios de exclusión: no aceptaron inclusión, niveles altos de natural killer, factor de necrosis tumoral y con enfermedades autoinmunes.

Las características clínicas de la gestante (edad, peso, talla, temperatura, saturación de $\mathrm{O}_{2}$, presión sanguínea [mmHg], frecuencia cardiaca y respiratoria) y datos del recién nacido (edad gestacional, peso y talla), se reportan con media \pm desviación estándar (DE) y se analizaron empleando la t de Student; por otra parte, las trombofilias, cesáreas, partos y abortos espontáneos se reportan en porcentajes y se analizaron empleando chi cuadrada, utilizando el paquete estadístico SPSS versión 25.

\section{Resultados}

Se incluyeron 29 mujeres para atención obstétrica, de las cuales 9 se incorporaron en grupo COVID-19 y 20 en grupo CONTROL. Comparando grupos, no se observó diferencia en datos generales y signos clínicos de embarazadas (peso, talla, índice de masa corporal, temperatura, saturación de $\mathrm{O}_{2}$, presión sanguínea, frecuencia cardiaca y respiratoria) (Tabla 1).

Con respecto al grupo COVID-19, el día del parto las pacientes 2, 4, 5, 6, 8 y 9 se encontraban entre día 0 y 10.5 de la infección, después de dar positivas por técnica de RT-PCR y únicamente las pacientes 1 , 3 y 7 habían superado la fase de resguardo recomendada para esta infección (14 días) (Fig. 1).

En cuanto al tipo de parto, hubo mayor prevalencia de COVID-19 en cesáreas vs. control (77.7 vs. 30\%) (Tablas 2 y 3). Se encontraron complicaciones en COVID19 como parto pretérmino (33.3\%), desprendimiento 


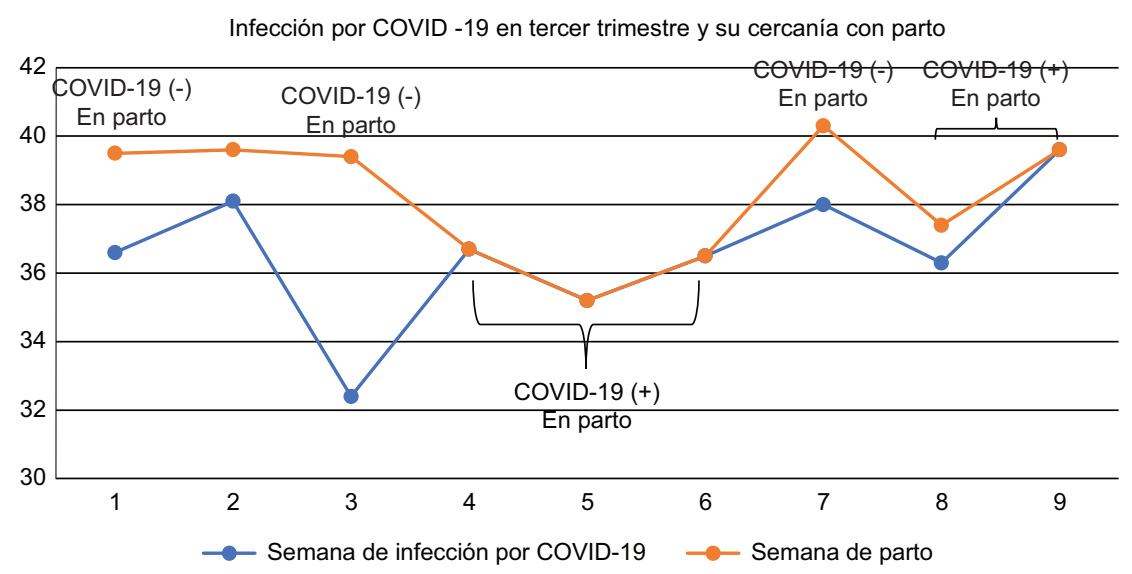

Figura 1. Muestra, semanas de gestación y parto en relación con el estado positivo o negativo de COVID-19. COVID-19: enfermedad por coronavirus 2019.

Tabla 1. Datos generales y signos maternos en día de parto Control $(\bar{x} \pm D E) \quad \operatorname{CoV} I D-19(\bar{x} \pm D E) \quad p \leq 0.05$ N 20 9

\begin{tabular}{|l|c|c|}
\hline Peso $(\mathrm{kg})$ & $72.9 \pm 11.1$ & $74.5 \pm 9.3$ \\
\hline Talla $(\mathrm{cm})$ & $1.62 \pm 0.04$ & $1.61 \pm 0.04$ \\
\hline IMC & $27.6 \pm 3.3$ & $28.5 \pm 2.5$ \\
\hline Edad (años) & $35.8 \pm 5$ & $35.4 \pm 4.6$ \\
\hline $\begin{array}{l}\text { Temperatura } \\
\left({ }^{\circ} \mathrm{C}\right)\end{array}$ & $36.4 \pm 0.3$ & $36.5 \pm 0.5$ \\
\hline $\begin{array}{l}\text { Saturación } \\
\text { de O }\end{array}$ & $95.1 \pm 0.7$ & $95.9 \pm 0.8$ \\
\hline $\begin{array}{l}\text { Presión } \\
\text { sanguínea } \\
\text { (mmHg) }\end{array}$ & $1.5 \pm 0.1$ & $1.5 \pm 0.1$ \\
\hline $\begin{array}{l}\text { Frecuencia } \\
\text { cardiaca }\end{array}$ & $81.8 \pm 13.6$ & $86.4 \pm 8.9$ \\
\hline $\begin{array}{l}\text { Frecuencia } \\
\text { respiratoria }\end{array}$ & $19.7 \pm 1.3$ & $18.7 \pm 8.4$ \\
\hline
\end{tabular}

respiratoria

DE: desviación estándar; IMC: índice de masa corporal.

prematuro de placenta $(20 \%)$, hipertensión $(11.1 \%)$, proteinuria $(11.1 \%)$, preeclampsia leve $(11.1 \%)$, oligohidramnios e hipomotilidad fetal (11.1\%); y en el grupo control de desprendimiento de placenta $(5 \%)$ y ruptura prematura de membranas (5\%) (Tablas 2 y 3). En control, las cesáreas fueron petición de la paciente (10\%) debido a segmento uterino delgado, inminencia de ruptura uterina y desprendimiento de placenta. Adicionalmente, la prevalencia de partos fue menor en el grupo COVID-19 vs. control (22.2 vs. 70\%) (Tablas 2 y 3). Ningún grupo presentó complicaciones en puerperio.
De acuerdo con los antecedentes obstétricos de cada paciente, el grupo COVID-19 presentó prevalencia de pérdida gestacional recurrente del $44.4 \%$, una paciente refirió dos abortos inducidos (11.1\%); en cuanto al control, la prevalencia de pérdida gestacional recurrente fue del 10\% (Tablas 2 y 3 ).

Los datos perinatales mostraron disminución estadística de semanas de gestación en COVID-19 comparando con control ( $37.8 \pm 1.8$ vs. $39 \pm 0.8 ; p \leq 0.05)$; con resultados similares en ambos grupos respecto al peso del recién nacido $(2,915 \pm 535.4$ vs. 3,013 \pm 518.8 gramos) y la talla $(48.1 \pm 2$ vs. $48.5 \pm 3.3 \mathrm{~cm})$ (Fig. 2).

En reportes histopatológicos placentarios, encontramos que COVID-19 vs. control presentó mayor prevalencia de trombos organizados en vellosidades placentarias ( 55.5 vs. $0 \%$ ); además, de trombo laminado en una arteria (11.1 vs. $0 \%$ ), hemorragia perivascular (33.3 vs. $0.0 \%$ ), hemorragia en vellosidades (55.5 vs. $42.1 \%$ ), hemorragia subcorial (11.1 vs. $5.2 \%$ ), hematoma retroplacentario (22.5 vs. $15.7 \%$ ) y edema estromal (44.4 vs. 31.5\%) (Fig. 3); en control fueron mayores los cambios isquémicos de vellosidades (84.2 vs. $55.5 \%$ ) y presencia de fibrina en espacios intervellosos (68.4 vs. 11.1\%) (Fig. 3). Se presentan los datos histopatológicos de las alteraciones en las 9 placentas (Fig. 4).

\section{Discusión}

Antecedentes de algunos patógenos virales (citomegalovirus, virus de la varicela zóster, etc.), generan inquietud de probables efectos del SARS-CoV-2 en embarazadas y el desarrollo del bebé17,18. 
Tabla 2. Características de parto (grupo COVID-19)

\begin{tabular}{|c|c|c|c|c|c|}
\hline N & Edad (años) & $\begin{array}{l}\text { Historial de } \\
\text { nacimientos }\end{array}$ & SDG & $\begin{array}{l}\text { Tipo de } \\
\text { alumbramiento }\end{array}$ & Características y complicaciones \\
\hline 1 & 38 & G2P1C1 & 39.5 & Vaginal & Sin complicaciones \\
\hline 2 & 35 & G0 & 36.6 & Vaginal & Sin complicaciones \\
\hline 3 & 36 & G0 & 39.4 & Cesárea & Desprendimiento de placenta \\
\hline 4 & 41 & G2A2 & 36.7 & Cesárea & Parto pretérmino \\
\hline 5 & 39 & G11A3P8 & 35.2 & Cesárea & Parto pretérmino \\
\hline 6 & 32 & G8A6C2 & 36.5 & Cesárea & Parto pretérmino \\
\hline 7 & 34 & G2C2 & 40.3 & Cesárea & Desprendimiento de placenta \\
\hline 8 & 44 & G2A2 (electivas) & 37.4 & Cesárea & Hipertensión, proteinuria, preeclampsia leve \\
\hline 9 & 27 & G3A3 & 39.6 & Cesárea & Oligohidramnios y hipomotilidad de bebe \\
\hline Total & $\begin{array}{l}35.8 \pm 5 \\
(\bar{x} \pm D E)\end{array}$ & - & $\begin{array}{c}37.8 \pm 1.8 \\
(\bar{x} \pm D E)\end{array}$ & $\begin{array}{l}77.7 \% \\
\text { cesárea }\end{array}$ & $\begin{array}{l}22.2 \% \text { sin complicaciones, } 33.3 \% \text { parto pretérmino, } 22.2 \% \\
\text { desprendimiento de placenta, } 11.1 \% \text { oligohidramnios, } 11.1 \% \\
\text { hipomotilidad, } 11.1 \% \text { hipertensión, } 11.1 \% \text { proteinuria y } 11.1 \% \\
\text { preeclampsia leve }\end{array}$ \\
\hline
\end{tabular}

COVID-19: enfermedad por coronavirus 2019; SDG: semanas de gestación; DE: desviación estándar.

\section{Tabla 3. Características de parto (control)}

\begin{tabular}{|c|c|c|c|c|c|}
\hline $\mathbf{N}$ & Edad (años) & $\begin{array}{l}\text { Historial de } \\
\text { nacimientos }\end{array}$ & SDG & $\begin{array}{l}\text { Tipo de } \\
\text { alumbramiento }\end{array}$ & Características y complicaciones \\
\hline 1 & 34 & G3P3 & 37.3 & Vaginal & Sin complicaciones \\
\hline 2 & 44 & G1A1 & 38.3 & Cesárea & Electivo, sin complicaciones \\
\hline 3 & 42 & G0 & 39.3 & Vaginal & Sin complicaciones \\
\hline 4 & 31 & G0 & 40.2 & Vaginal & Sin complicaciones \\
\hline 5 & 39 & G1P1 & 38.5 & Vaginal & Sin complicaciones \\
\hline 6 & 36 & G1A1 & 40.3 & Vaginal & Sin complicaciones \\
\hline 7 & 32 & G0 & 38.6 & Cesárea & Desprendimiento de placenta \\
\hline 8 & 36 & G1C1 & 38.2 & Cesárea & Electivo, sin complicaciones \\
\hline 9 & 31 & G1P1 & 38.5 & Vaginal & Sin complicaciones \\
\hline 10 & 35 & G0 & 40.2 & Vaginal & Sin complicaciones \\
\hline 11 & 39 & G1C1 & 39.5 & Cesárea & Segmento uterino delgado y sin complicaciones \\
\hline 12 & 37 & G1P1 & 38.6 & Vaginal & Sin complicaciones \\
\hline 13 & 38 & G2C1A1 & 39.5 & Cesárea & Segmento uterino delgado y ruptura prematura de membranas \\
\hline 14 & 36 & G0 & 39.4 & Vaginal & Sin complicaciones \\
\hline 15 & 43 & G3A3 & 39.1 & Cesárea & Segmento uterino delgado y sin complicaciones \\
\hline 16 & 40 & G0 & 39.2 & Vaginal & Sin complicaciones \\
\hline 17 & 34 & G0 & 38.2 & Vaginal & Sin complicaciones \\
\hline 18 & 29 & G0 & 40.2 & Vaginal & Sin complicaciones \\
\hline 19 & 32 & G0 & 39.4 & Vaginal & Sin complicaciones \\
\hline 20 & 27 & G1P1 & 39.2 & Vaginal & Sin complicaciones \\
\hline Total & $\begin{array}{l}35.4 \pm 4.6 \\
(\bar{x} \pm D E)\end{array}$ & - & $\begin{array}{l}39 \pm 0.8 \\
(\bar{x} \pm D E)\end{array}$ & $\begin{array}{l}30 \% \\
\text { cesáreas }\end{array}$ & $\begin{array}{l}90 \% \text { sin complicaciones, } 5 \% \text { desprendimiento de placenta y } 5 \% \\
\text { desprendimiento de membrana }\end{array}$ \\
\hline
\end{tabular}


A

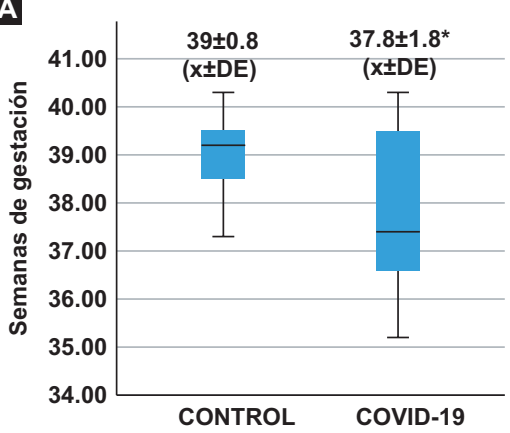

B

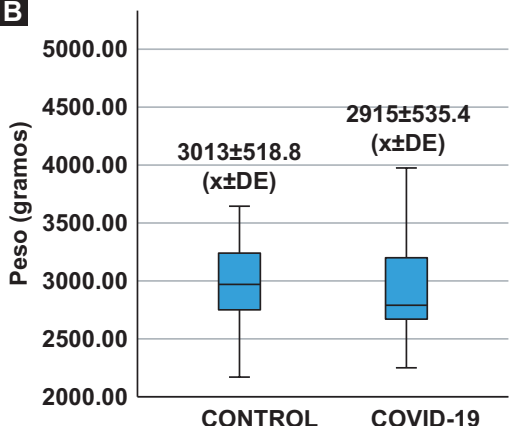

C

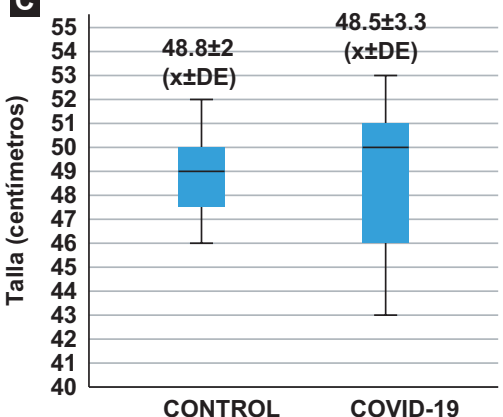

Figura 2. Datos perinatales de recién nacidos de madres infectadas por COVID-19 durante el último trimestre de embarazo. A: semanas de gestación. B: peso del recién nacido. C: talla de recién nacido.

${ }^{*}$ Diferencia estadística de semanas de gestación (control vs. COVID-19, $p \leq 0.05$ ), $t$ de Student.

COVID-19: enfermedad por coronavirus 2019; DE: desviación estándar.

Patología placentaria (COVID-19 vs. control)

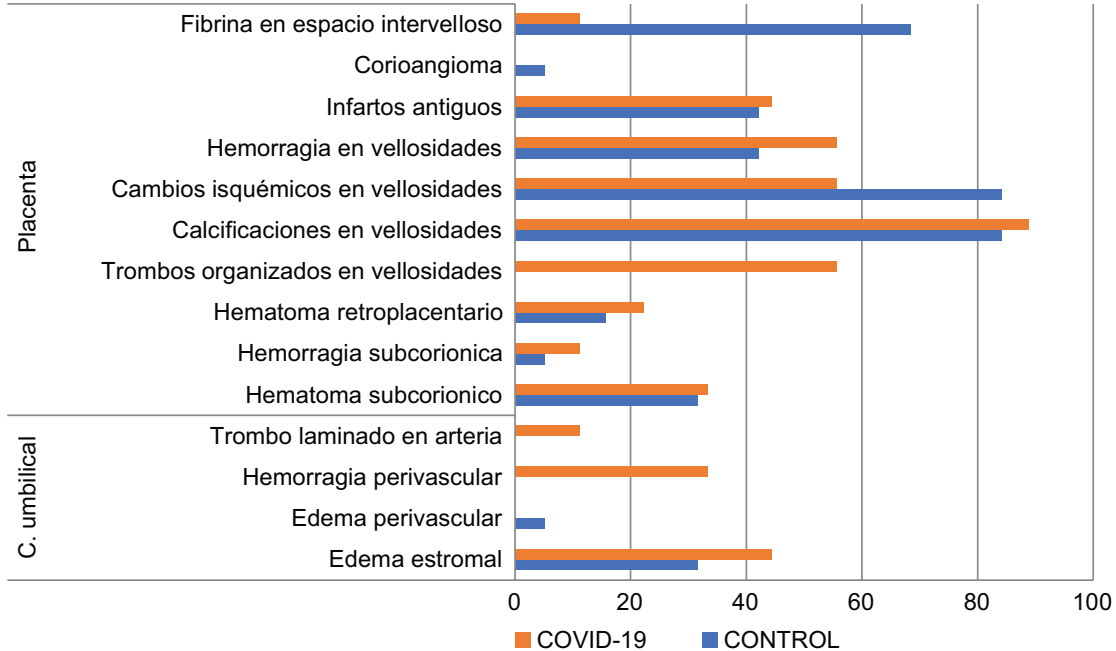

Figura 3. Diferencia estadística de fibrina en espacio intervelloso (COVID-19 vs. control, $p \leq 0.05)$, chi cuadrada.
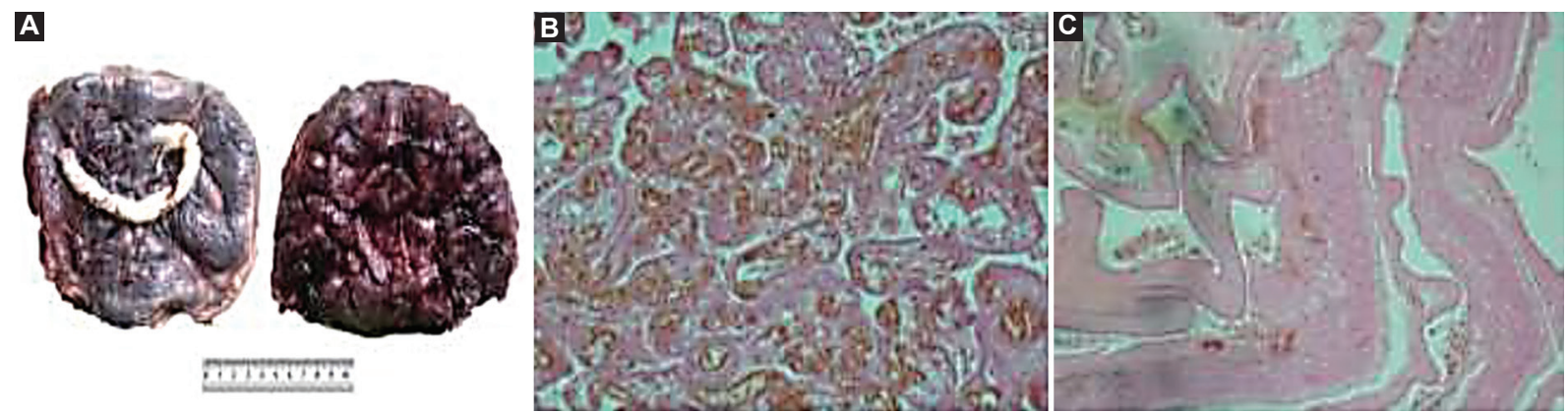

Figura 4. A: descripción macroscópica: placenta de $405 \mathrm{~g}$, áreas hemáticas con hematoma de $5 \mathrm{~cm}$. B y C: descripción microscópica: hematoma retroplacentario marginal de $5 \mathrm{~cm}$, trombos organizados no laminados, vellosidades coriónicas del tercer trimestre con calcificaciones en el $5 \%$ de la superficie y cambios isquémicos en el $10 \%$. Similares a las otras 8 placentas estudiadas.

En relación con el SARS-CoV y MERS, se ha reportado incremento de aborto de primer trimestre, parto pretérmino, ruptura prematura de membranas, restricción de crecimiento intrauterino y tasa de cesárea ${ }^{19-23}$, con histopatología placentaria que reporta gran cantidad de fibrina (intervellosa y 
subcoriónica), calcificaciones en vellosidades, infartos, hipotrofia placentaria y vasculopatía trombótica en algunas vellosidades ${ }^{24-32}$. SARS-CoV-2, SARSCoV y MERS-CoV tienen mecanismos equivalentes de infección, lo que quizás resulte en alteraciones materno/fetales similares ${ }^{33}$.

Por su parte, no existe evidencia concluyente de transmisión vertical intrauterina del SARS-CoV-2 y las complicaciones maternas/fetales reportadas han incluido sufrimiento fetal intrauterino $(14 \%)$ y ruptura prematura de membranas $(8 \%)$ y en los recién nacidos, dificultad respiratoria (6\%), síntomas gastrointestinales $(4 \%)$ y fiebre $(3 \%)^{34}$. En este estudio, las pacientes positivas a COVID-19 no desarrollaron síndrome respiratorio agudo severo, manteniendo estado latente y quizás compensado inmunitariamente ${ }^{35}$. Se observaron complicaciones durante el alumbramiento, como: alta tasa de cesárea (77\%), parto pretérmino (33.3\%), desprendimiento de placenta $(22.2 \%)$, preeclamsia leve (11.1\%) y asociación de oligohidramnios e hipomotilidad fetal (11.1\%), coincidiendo con publicaciones de embarazos con COVID-19 severa, los cuales presentaron altas tasas de cesárea, de hasta el $92 \%$, y parto pretérmino del 11 al $42 \%{ }^{35-37}$, que presentaron comorbilidades o complicaciones como preeclampsia, diabetes gestacional, hipotiroidismo, placenta previa y cirugías uterinas previas ${ }^{38}$. A su vez, la COVID-19 se ha localizado predominantemente en células del sincitiotrofoblasto en la interfaz maternofetal de la placenta ${ }^{38}$. Además, en placentas de semana 27 y 39 obtenidas de mujeres infectadas por SARSCoV-2 severo se ha encontrado incremento de factores que predisponen a alteraciones placentarias, como trombosis, infartos y remodelación de la pared vascular en vellosidades coriónicas ${ }^{39}$.

En nuestros datos perinatales se observa que embarazadas asintomáticas con COVID-19 en tercer trimestre presentan disminución de semanas de gestación en comparación con control $(37.8 \pm 1.8$ vs. 39 $\pm 0.8 ; p=0.05)$. De igual forma, Metz (2021), Flores (2021) y Martínez (2020) encontraron en pacientes con síntomas severos disminución de semanas de gestación y bajo peso al nacer ${ }^{40-42}$.

A su vez, este trabajo muestra incremento de trombos organizados en vellosidades (33.3 vs. $0 \%$ ) y hemorragia perivascular (55.5 vs. $0 \%$ ) en COVID19 vs. control, similar a estudios que reportan infartos de vellosidades ( 20 vs. $2 \%$ ), necrosis fibrinoide (20 vs. $0 \%$ ), hipertrofia mural de membranas (33 vs. $4 \%$ ), corangiosis ( 27 vs. $5 \%$ ), edema velloso ( 27 vs. $9 \%$ ), trombos intravellosos (40 vs. $8 \%$ ), fibrina (52 vs. $6 \%$ ), microcalcificaciones (60 vs. $4 \%$ ), pequeñas vellosidades fibróticas (28 vs. $0 \%$ ), aglutinación de vellosidades (18 vs. $0 \%$ ) y aumento de nudos sincitiales (40 vs. $2 \%)^{38,42}$.

El estado de hipercoagulación que se presenta durante el embarazo podría favorecer estas alteraciones, aunado a la alta prevalencia de trombofilias heredadas que presenta la población mexicana ${ }^{43,44}$, donde se ha observado un estado protrombótico inducido por el incremento en sangre periférica del dímero D, del 36 al $43 \%$ en pacientes con COVID-1945.

El estado protrombótico por COVID-19 en embarazadas quizás sea generado de forma similar a los eventos reportados de trombosis venosa profunda, embolia pulmonar, infarto de miocardio y accidente cerebrovascular isquémico, en pacientes con COVID-1946,47.

En este estudio reportamos pocos casos de mujeres asintomáticas que presentaron la prueba de COVID-19 positiva, al final del embarazo; lo cual indica que puede existir ya riesgo perinatal, por medio del estudio histopatológico de la placenta, en mujeres que no desarrollaron el síndrome respiratorio agudo severo; se requiere mayor investigación conforme se presenten más casos de embarazo con COVID-19 para identificar qué pacientes asintomáticas positivas realmente tienen riesgo perinatal.

La salud de las mujeres embarazadas, independientemente de que presenten síntomas leves con mayor frecuencia, incluso cursen asintomáticas, como fueron nuestras pacientes; requiere vigilancia estrecha durante esta pandemia y asistencia crítica oportuna en casos severos.

\section{Limitaciones}

El tamaño pequeño de la muestra, que por sí solo no es significativo, pero aunado a estudios que se han desarrollado hasta la fecha puede dar información que nos ayude a detectar alteraciones que pudieran ser generadas en el parto por la COVID-19.

\section{Conclusiones}

La infección asintomática por COVID-19 en el tercer trimestre del embarazo puede potenciar un perfil protrombótico preexistente, incrementando el riesgo de trombosis; principalmente si se desarrolla el síndrome respiratorio agudo severo, generando alteraciones como desprendimiento de placenta, preeclampsia, hemorragias e incremento de partos pretérmino. 


\section{Financiamiento}

La presente investigación no ha recibido ninguna beca específica de agencias de los sectores públicos, comercial o sin ánimo de lucro.

\section{Conflicto de intereses}

Los autores declaran no tener conflicto de intereses.

\section{Responsabilidades éticas}

Protección de personas y animales. Los autores declaran que para esta investigación no se han realizado experimentos en seres humanos ni en animales.

Confidencialidad de los datos. Los autores declaran que han seguido los protocolos de su centro de trabajo sobre la publicación de datos de pacientes.

Derecho a la privacidad y consentimiento informado. Los autores declaran que en este artículo no aparecen datos de pacientes.

\section{Bibliografía}

1. Su S, Wong G, Shi W, Liu J, Lai ACK, Zhou J, et al. Epidemiology, genetic recombination, and pathogenesis of coronaviruses. Trends Microbiol. 2016;24(6):490-502.

2. Cui J, Li F, Shi Z. Origin and evolution of pathogenic coronaviruses. Nat Rev Microbiol. 2019;17:181-92.

3. Aragón R, Vargas I, Miranda M. COVID-19 por SARS-CoV-2: la nueva emergencia de salud. Rev Mex Pediatr. 2020;86(6):213-8

4. Li Q, Guan X, Wu P, Wang X, Zhou L, Tong Y, et al. Early transmission dynamics in Wuhan, China, of novel coronavirus-infected pneumonia. N Engl J Med. 2020;382(13):1199-207.

5. Wan Y, Shang J, Graham R, Baric RS, Li F. Receptor recognition by the novel coronavirus from Wuhan: An analysis based on decade-long structural studies of SARS coronavirus. J Virol. 2020:94(7):e00120-27.

6. José J Elizalde-González. SARS-CoV-2 and COVID-19. A pandemic review- Med Crit 2020;34(1):53-67 doi: 10.35366/93281.

7. Collange O, Tacquard $C$, Delabranche $X$, Leonard-Lorant I, Ohana M,Onea M, et al. Coronavirus Disease 2019: Associated Multiple Organ Damage. Open Forum Infect Dis. 2020;7(7):ofaa249. doi:10.1093/ofid/ofaa249

8. Yang M, Chen S, Huang B, Zhong JM, Su H, Chen YJ, et al. Pathological findings in the testes of COVID-19 patients: Clinical implications. Eur Urol Focus. 2020;1(20):30144-9.

9. Li R, Yin T, Fang F, Li Q, Chen J, Wang Y, et al. Potential risks of SARS-CoV-2 infection on reproductive health. Reprod Biomed Online. 2020;41(1):89-95.

10. Duque J, Duque D, Pelaez F. El COVID-19 también afecta el sistema nervioso por una de sus compuertas: el órgano vascular de la lámina terminal y el nervio olfatorio. Alerta neurológica, prueba de disosmia o anosmia puede ayudar a un diagnóstico rápido. Int J Odontostomat. 2020;14(3):285-7.

11. Iadecola C, Anrather, Kamel H. Effects of COVID-19 on the nervous system. Cell Press. 2020;183:6-28.

12. Carbonell A, Garcia A, Garcia O, Frías M, Cabrera MA. Trombosis y COVID-19: Atención primaria clave en el abordaje interdisciplinar. Semergen. 2020;46(S1):100-1.

13. Shabes E, Mithal B, Otero S, Azad HA, Miller ES, Goldstein JA.Placental pathology in COVID-19. Am J Clin Pathol. 2020;154(1):1-10.

14. Panahi L, Amiri M, Pouy S. Risks of novel coronavirus disease (COVID-19) in pregnancy; a narrative review. Arch Acad Emerg Med. 2020;8(1):e34

15. Johns Hopkins University \& Medicine. Coronavirus Resource Center [Internet]. Johns Hopkins University \& Medicine [último acceso: 28 diciembre 2020]. Disponible en: https://coronavirus.jhu.edu/map.html

16. Khan S, Jun L, Nawsherwan, Siddique R, Li Y, Han G, et al. Association of COVID-19 with pregnancy outcomes in health-care workers and general women. Clin Microbiol Infect. 2020;26:788-90.
17. Coyne $\mathrm{C}$, Lazear H. Zika virus - reigniting the TORCH. Nat Rev Microbiol. 2016:14(11):707-15

18. Wastnedge E, Reynolds R, van Boeckel S, Stock SJ, Denison FC, Maybin JA, et al. Pregnancy and COVID-19. Physiol Rev. 2021;101:303-18.

19. Yudin M, Steele D, Sgro M, Read SE, Kopplin P, Gough KA. Severe acute respiratory syndrome in pregnancy. Obstet Gynecol. 2005;105:124-7.

20. Wong S, Chow K, Leug T. Pregnancy and perinatal outcomes of women with severe acute respiratory syndrome. Obstet Gynecol. 2004;191:292-7.

21. Lam C, Wong S, Leung T. A case-controlled study comparing clinical course and outcomes of pregnant and non-pregnant women with severe acute respiratory syndrome. BJOG. 2004;111:171-4.

22. Robertson C, Lowther S, Birch T. SARS and pregnancy: a case report. Emerg Infect Dis. 2004;10:345-8.

23. Schneider E, Duncan D, Reiken M, Perry R, Messick J, Sheedy C, et al. SARS in pregnancy. AWHONN Lifelines. 2004:8(2):122-8. doi:10.1177/1091592304265557

24. Alfaraj S, Al-Tawfiq J, Memish Z. Middle East respiratory syndrome coronavirus (MERSCoV) infection during pregnancy: report of two cases and review of the literature. J Microbiol Immunol Infect. 2019:52:501-3.

25. Jeong S, Sung S, Sung J, Ahn SY, Kang ES, Chang YS. MERS-CoV infection in a pregnant woman in Korea. J Korean Med Sci. 2017;32: 717-20.

26. Alserehi $\mathrm{H}$, Wali $\mathrm{G}$, Alshukairi A, Alraddadi B. Impact of Middle East respiratory syndrome coronavirus (MERS-CoV) on pregnancy and perinatal outcome. BMC Infect Dis. 2016;16:105.

27. Assiri A, Abedi G, Al Masri M, Bin Saeed A, Gerber SI, Watson JT. Middle East respiratory syndrome coronavirus infection during pregnancy: a report of 5 cases from Saudi Arabia. Clin Infect Dis. 2016;63:951-3.

28. Malik A, El Masry K, Ravi M, Sayed F. Middle East respiratory syndrome coronavirus during pregnancy, Abu Dhabi, United Arab Emirates, 2013. Emerg Infect Dis. 2016;22:515-7.

29. Park M, Kim H, Choi D, Sung JH, Kim JH. Emergency cesarean section in an epidemic of the middle east respiratory syndrome: a case report. Korean J Anesthesiol. 2016;69:289-91.

30. Payne D, Iblan I, Alqasrawi S. Stillbirth during infection with Middle East respiratory syndrome coronavirus. J Infect Dis. 2014;209:1870-2.

31. Sharps M, Hayes D, Lee S, Zou Z, Brady CA, Almoghrabi Y, et al. A structured review of placental morphology and histopathological lesions associated with SARS-CoV-2 infection. Placenta. 2020;101:13-29.

32. Ng W, Wong S, Lam A, Mak YF, Yao H, Lee KC, et al. The placentas of patients with severe acute respiratory syndrome: a pathophysiological evaluation. Pathology. 2006;38(6):210-8.

33. Wong S, Chow K, Leung T, Ng WF, Ng TK, Shek CC, et al. Pregnancy and perinatal outcomes of women with severe acute respiratory syndrome. Am J Obstet Gynecol. 2004;91(1):292-7.

34. Oliva J. SARS-CoV-2: origen, estructura, replicación y patogénesis. Alerta. 2020:3(2):79-86.

35. Akhtar H, Patel C, Abuelgasim E, Harky A. COVID-19 (SARS-CoV-2) infection in pregnancy: A systematic review. Gynecol Obstet Invest. 2020;85(4):295-306

36. Della Gatta A, Rizzo R, Pilu G, Simonazzi G. Coronavirus disease 2019 during pregnancy: a systematic review of reported cases. Am J Obstet Gynecol. 2020;223:36-41

37. Zaigham M, Andersson O. Maternal and perinatal outcomes with COVID-19: A systematic review of 108 pregnancies. Acta Obstet Gynecol Scand. 2020;99(7):823-9.

38. Mendoza M, Garcia I, Maiz N, Rodo C, Garcia-Manau P, Serrano B, et al. Preleclampsianlike syndrome induced by severe COVIDa19: a prospective observational study. BJOG. 2020;127(11):1374-80.

39. Singh N, Buckley T, Shertz W. Placental pathology in COVID-19: Case series in a community hospital setting. Cureus. 2020;13(1):e12522.

40. Flores A, Miranda J, Vega S, Valdespino Y, Helguera C, Espejel A, et al. Molecular insights into the thrombotic and microvascular injury in placental endothelium of women with mild or severe COVID-19. Cells. $2021 ; 10(364): 1-21$.

41. Martinez O, Vouga M, Cruz S, Forcen Acebal L, Panchaud A, Muñoz-Chápuli $\mathrm{M}$, et al. Association between mode of delivery among pregnant women with COVID-19 and maternal and neonatal outcomes in Spain. JAMA. 2020;324(3):296-9.

42. Metz T, Clifton R, Huges B, Sandoval G, Saade GR, Grobman WA, et al. Disease severity and perinatal outcomes of pregnant patients with coronavirus disease 2019 (COVID-19). Obstet Gynecol. 2021;137(4):571-80.

43. Shanes E, Mithal L, Otero S, Azad HA, Miller ES, Goldstein JA.Placental pathology in COVID-19. Am J Clin Pathol. 2020;154:23-32.

44. Almagro D. La hemostasia en el embarazo. Revista Cubana de Hematología, Inmunología y Hemoterapia. 2000;16(2):90-8.

45. Lujan J, Durand C, Avila F, Rebollar D. Incidence of hereditary thrombofilies in a population of Mexican women. Obstetrics \& Gynecology International Journal. 2020;11(4):208-13.

46. Lippi G, Favaloro E. D-dimer is associated with severity of coronavirus disease 2019: A pooled analysis. Thromb Haemost. 2020;120(5):876-7.

47. Violi F, Pastori D, Cangemi R, Pignatelli P, Loffredo L. Hypercoagulation and antithrombotic treatment in coronavirus 2019. Thromb Haemost. 2020;120(6):949-56 